\title{
Application Article \\ Improved Low-Profile Helical Antenna Design for INMARSAT Applications
}

\author{
Shiqiang Fu, Yuan Cao, Yue Zhou, and Shaojun Fang \\ School of Information Science and Technology, Dalian Maritime University, Liaoning, Dalian 116026, China \\ Correspondence should be addressed to Shiqiang Fu, fushq@dlmu.edu.cn
}

Received 9 April 2012; Revised 19 May 2012; Accepted 20 May 2012

Academic Editor: Z. Chen

Copyright () 2012 Shiqiang Fu et al. This is an open access article distributed under the Creative Commons Attribution License, which permits unrestricted use, distribution, and reproduction in any medium, provided the original work is properly cited.

A new low-profile variable pitch angle cylindrical helical antenna employing a copper strip as impedance transformer is proposed in this paper. Under the circumstance of a limited antenna height, the circular polarization performance of the antenna has been enhanced by changing the pitch angle and the input impedance matching has been improved by adjusting the copper strip match stub. The design method of the proposed antenna is given. The optimal antenna structure for INMARSAT application has been fabricated and measured. The measured results show that in the whole maritime satellite communication work band the VSWR is less than 1.2, its antenna gain is higher than $9 \mathrm{dBi}$, and the axial ratio is lower than $2.5 \mathrm{~dB}$. The experimental results have a good agreement with the simulations. The proposed antenna is compact and easy tuning. It provides a promising antenna element for maritime satellite communication applications.

\section{Introduction}

Helical antennas have been known for a long time [1]. Axialmode helical antennas are of considerable interest in satellites and radars because they provide circular polarization over a wide bandwidth without the need for a polarizer. In the past, the axial-mode helical antennas continue to appear in new designs and research papers. Numerous modifications focus on optimizing the length, pitch angle or radius of the helical antenna for a good radiation performance [25]. For example, in [2], Professor Nakano et al. calculated the helical arm length current distribution and realized an extremely low-profile cylindrical helix as a radiating element of circular polarization by the combination of low pitch and a small number of turns. In [3], exponential pitch spacing was proposed in order to increase the CP bandwidth of the axial-mode helix. In [4], an elliptical helical antenna with a variable pitch angle was presented in the pursuit of improved circular polarization as well as its directivity. In [5], a robust and low-profile hemispherical helical antenna was introduced which produces very pure circular polarization radiation over a broader angular range.

The purpose of this paper is to call attention to the fact that the antenna size is restricted in practical designs. A new design method of the low-profile helical antenna is given with limited antenna height. Based on this method, a low-profile variable pitch angle cylindrical helical antenna employing a copper strip match stub is proposed. Kraus and Nakano have proposed some techniques to match the helical antenna to $50 \mathrm{ohms}$ in $[6,7]$. A more simple and effective self-matching scheme has been presented in [8] by adding a copper strip on the connecting wire as impedance transformer. However, the position of the copper strip is fixed at the center of the connecting wire and detailed matching scheme is not given.

In this paper, under the circumstance of a given antenna height, the performance of circular polarization of the antenna has been enhanced by changing the pitch angle, and the input impedance matching has been improved by adjusting the copper strip match stub size and position. In addition, a parametric study of the proposed antenna is presented to provide the engineers with information for designing, modifying, and optimizing such an antenna. And also, the simulated results are validated by experimental data at L-band for INMARSAT applications.

\section{Antenna Configuration and Design Approach}

Figure 1 shows the proposed antenna model configuration. The variable pitch angle helix conductor supported by 


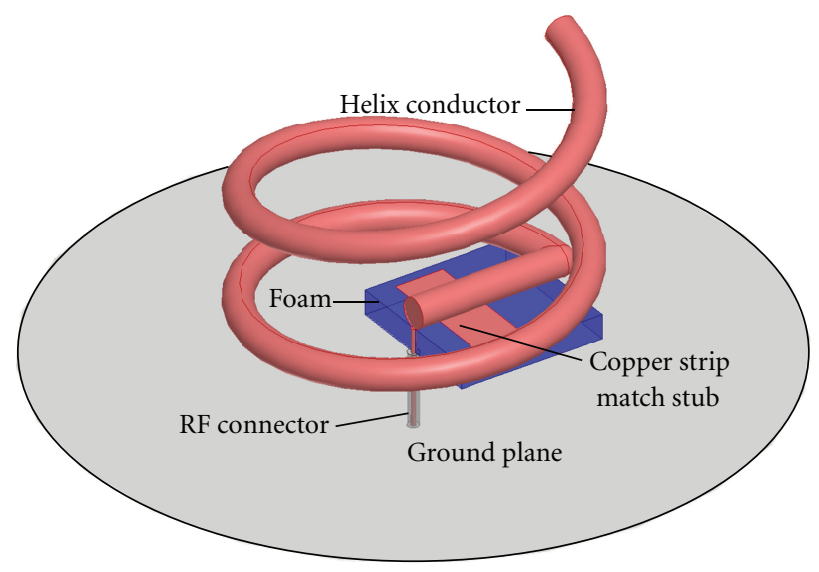

(a) Perspective view

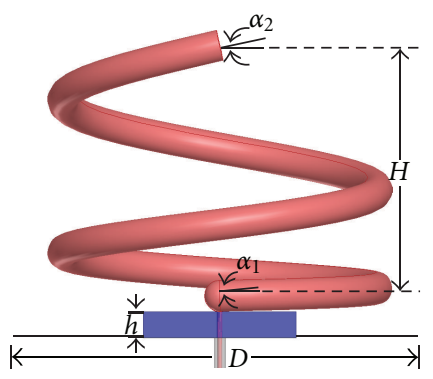

(b) Side view

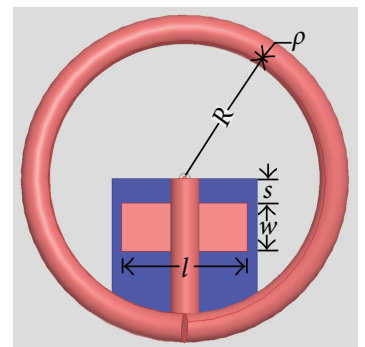

(c) Top view

Figure 1: Antenna model configuration.

expanded polystyrene (EPS) foam $\left(\varepsilon_{r} \approx 1.05\right)$ is mounted on a big ground plane. A thin copper strip was bonded to the center-fed helix conductor between the feed point and the beginning of the helix proper as suggested in Figure 1.

The configuration parameters are designated as follows: helical radius $R$, pitch angle $\alpha_{1}$ at the feed end, pitch angle $\alpha_{2}$ at the open end, helical height $H$, conductor wire radius $\rho$, total number of helical turns $N$, supported foam height $h$, copper strip position $s$, copper strip width $w$, and length $l$. The equations that describe the proposed helix conductor are

$$
\begin{gathered}
x=R \cdot \cos (\varphi), \quad y=R \cdot \sin (\varphi), \\
z=R \cdot \tan \left\{\left[\alpha_{1}+\left(\alpha_{2}-\alpha_{1}\right) \frac{\varphi}{2 \pi n}\right] \frac{\pi}{180}\right\}, \varphi
\end{gathered}
$$

where $x, y, z$ are the Cartesian coordinates. For a complete helix $0 \leq \varphi \leq 2 \pi N$ and for a truncated helix $\varphi$ can be used with $0 \leq \varphi \leq 2 \pi n$, where $n$ is the actual number of turns.

In practical designs, the antenna size is often limited, such as height and cross section. So to reduce the helix height sufficiently, the pitch spacing and pitch angle are varied as the $\alpha_{1}$ and $\alpha_{2}$ are varied. From [9], we can conclude some theories and experiences that are useful for optimizing the size of the helix, such as helical circumference $C$ that should be around $\lambda$ (the wavelength at a test frequency), and the pitch angle that should be in a range of $12^{\circ} \sim 14^{\circ}$. However, the pitch angle could be less than $7^{\circ}$ referring to the research made by Nakano et al. [2].
In our design, for a given antenna height $H \leq 5 \mathrm{~cm}$ demand, the pitch angle $\alpha_{2}=7^{\circ}$ will be decided according to (1). Assuming in accordance with the traditional design requirements $[9,10]$, the antenna helical turns will be $N \approx$ 1.2. The antenna circular polarization performance will be poor at this time. So the design method should be improved taking into account the limited antenna height. Based on previous studies, we set $N=2$ and $C=2 \pi R=\lambda$.

At first, the commercial simulation tool HFSS has been used to optimize $\alpha_{1}$ for the best circular polarization radiation performance at a given $\alpha_{2}=7^{\circ}$. The $\alpha_{1}$ can be lower than $\alpha_{2}$ (called positive variable pitch angle) or higher than $\alpha_{2}$ (called negative variable pitch angle). There will be optimized $\alpha_{1}$ for the optimal radiation performance, such as lower axial ratio as shown in Figure 2. And then the antenna input impedance can be easily adjusted to $50 \Omega$ by changing the copper strip match stub size and position under the premise of ensuring the optimal radiation performance. Throughout the studies, it is found that the real part of the antenna impedance can be easily controlled by mainly changing the copper strip position $s$, while the imaginary part of the antenna impedance can be adjusted by mainly changing the copper strip width $w$ and length $l$, which can be seen in Figure 3. The impedance matching can be achieved without major modification of the radiator, and the $\mathrm{CP}$ characteristic is also not so sensitive to the changing of the match stub. 


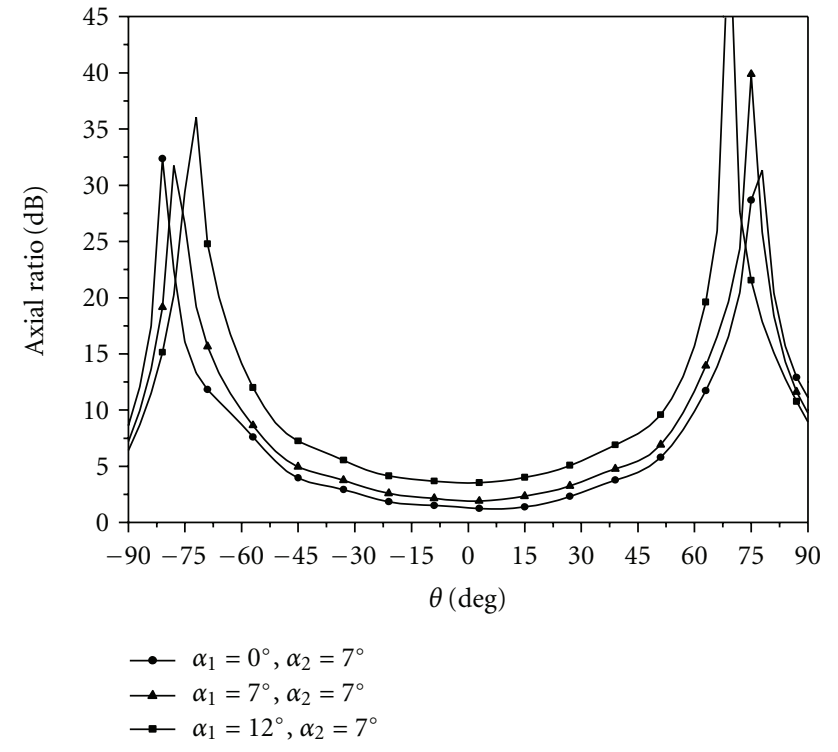

Figure 2: Wide angle axial ratio simulation results with variable pitch angle.

\section{Experimental Results and Discussions}

The antenna was optimized for operation in the INMARSAT working band $(1.525 \sim 1.660 \mathrm{GHz})$ with center frequency $1.593 \mathrm{GHz}$. After extensive simulations, the following values are selected: $R=30 \mathrm{~mm}, H=50 \mathrm{~mm}, \alpha_{1}=0^{\circ}, \alpha_{2}=7^{\circ}$, $\rho=2.5 \mathrm{~mm}, w=10 \mathrm{~mm}, l=25 \mathrm{~mm}, s=7 \mathrm{~mm}, h=$ $5 \mathrm{~mm}$, and $N=2$. The antenna is located at the center of circular copper plate ground plane with a diameter of $D=180 \mathrm{~mm}$. Figure 4 shows a photograph of the completed helical antenna prototype. A plastic rod is used to provide mechanical stability.

The experimental and simulated reflection characteristics of the antenna measured using Agilent N5230A network analyzer and Ansys HFSS, respectively, are compared in Figure 5. It can be seen that the measured results are found to reasonably agree with the simulated data. A good measured input impedance match characteristics of $S_{11}<-15 \mathrm{~dB}$ in the range of $1.5 \sim 1.8 \mathrm{GHz}$ is obtained. There exists a frequency offset between the measurements and simulations due to the assembly error. It can be adjusted by the copper strip match stub. The adjusted measured $S_{11}$ is less than $-20 \mathrm{~dB}$ over the INMARSAT working band, yielding to a VSWR $<1.2$.

Circular polarization radiation performance of the antenna has been measured using a rotating linearly polarized transmit horn antenna. The simulated and measured gain and axial ratio characteristics at the bore sight of the antenna are presented in Figure 6. As shown in Figure 6, this antenna possesses simulated $3 \mathrm{~dB}$ axial ratio bandwidth of $16 \%(1.47 \mathrm{GHz}$ to $1.72 \mathrm{GHz})$. The measured axial ratio value is less than $2.5 \mathrm{~dB}$ over the INMARSAT operating band, which is equivalent to on-axis cross-polarization discrimination (XPD) of at least $17 \mathrm{~dB}$. The gain of the antenna was measured at approximately $9 \mathrm{dBi}$ across the whole operating band. The measured gain is slightly lower than the simulated one. The radiation patterns of both

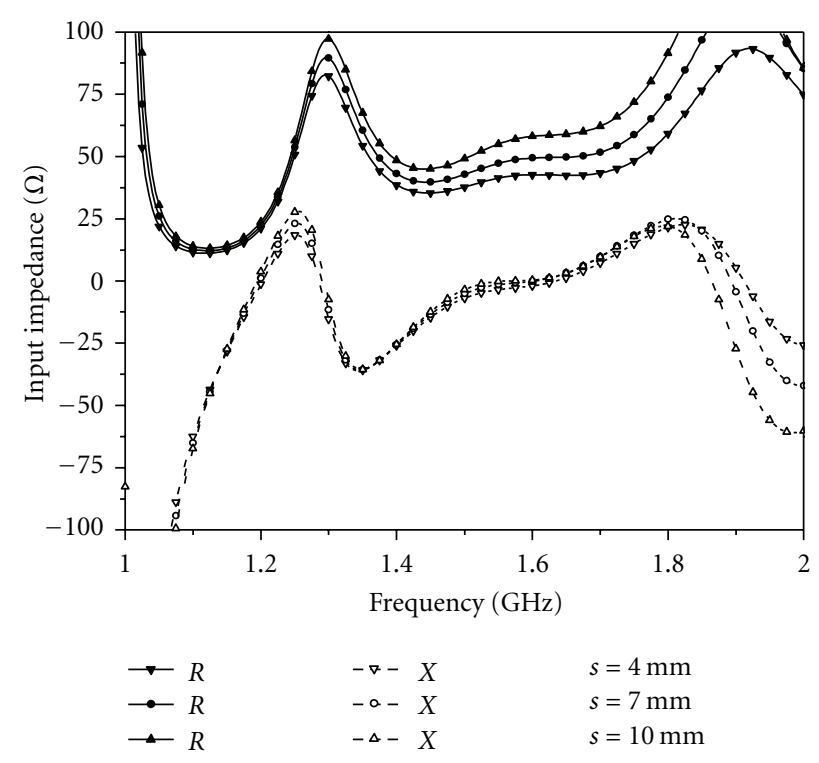

(a) Impedance curve as a function of $s$

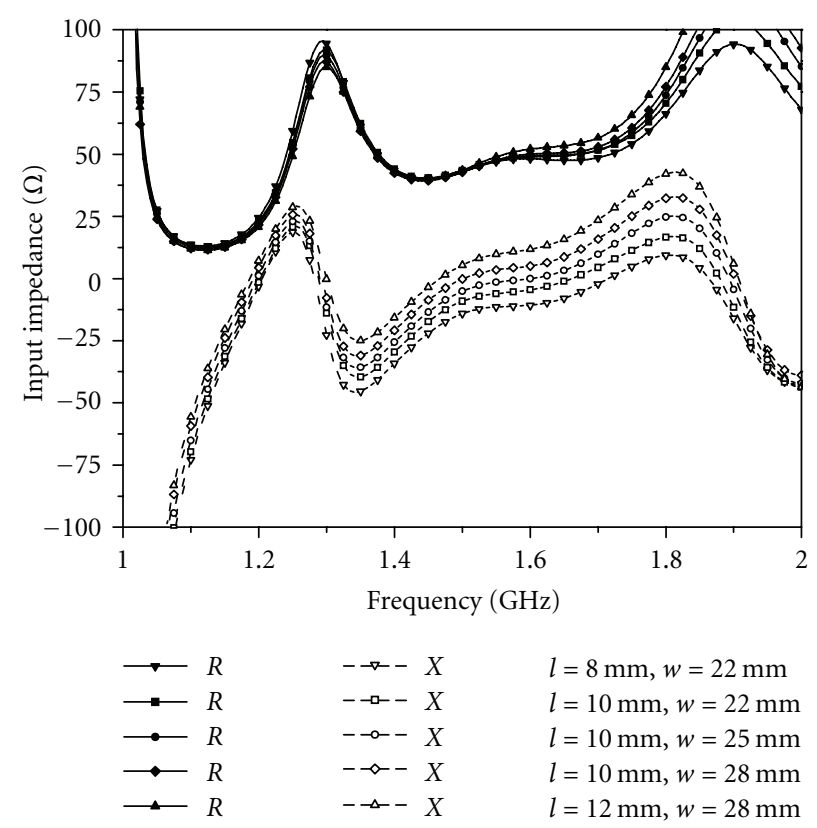

(b) Impedance curve as a function of $l$ and $w$

Figure 3: Effect of copper strip match stub position and size on input impedance.

RHCP and LHCP at the center frequency are shown in Figure 7 . The measured patterns are in good agreement with the computed ones. The discrepancy between them can be mainly attributed to fabrication and measurement errors.

\section{Conclusions}

A new improved low-profile helical antenna design for INMARSAT applications has been presented. The remarkable feature of the antenna is that it is very easy to design and tune with limited antenna height. Firstly, the circular polarization radiation performance of the antenna can be 


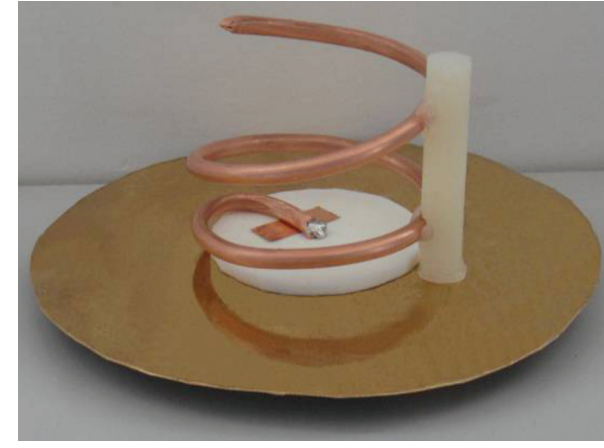

Figure 4: The fabricated antenna prototype.

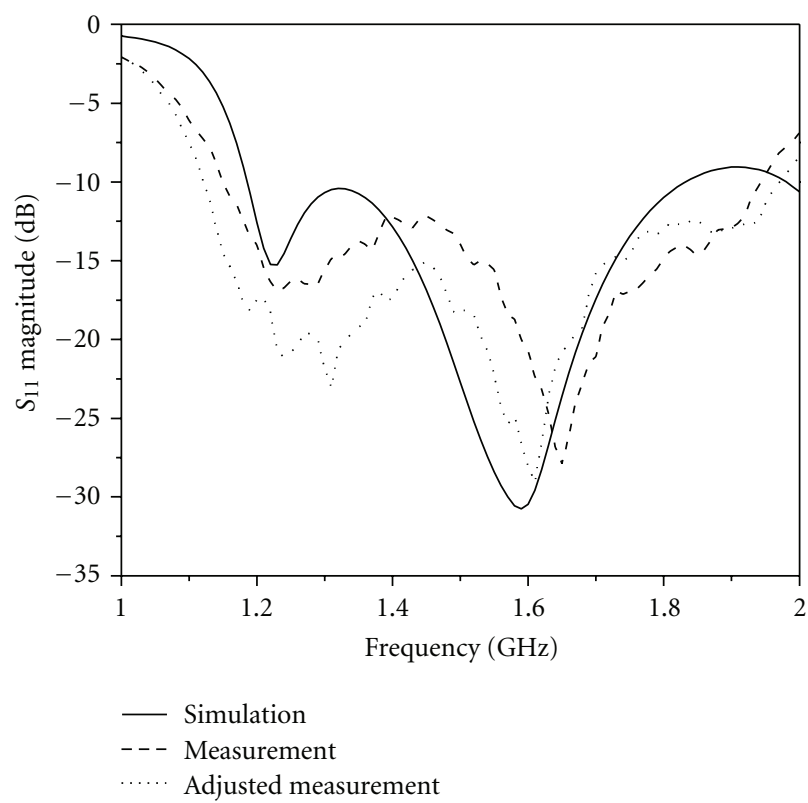

FIGURE 5: Simulated and measured reflection characteristics versus frequency.

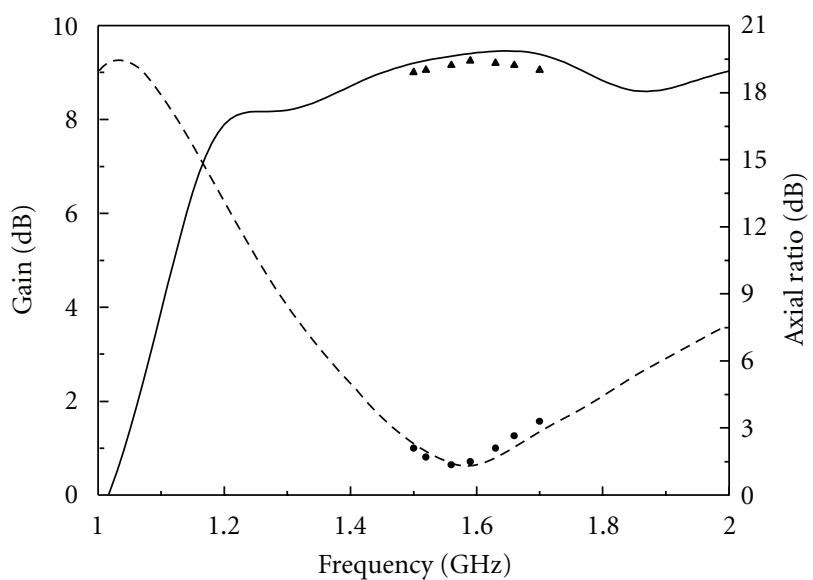

$\begin{array}{cccc}- & \text { Simulated gain } & -- & \text { Simulated AR } \\ \triangle & \text { Measured gain } & - & \text { Measured AR }\end{array}$

Figure 6: Simulated and measured gain and axial ratio at the boresight.

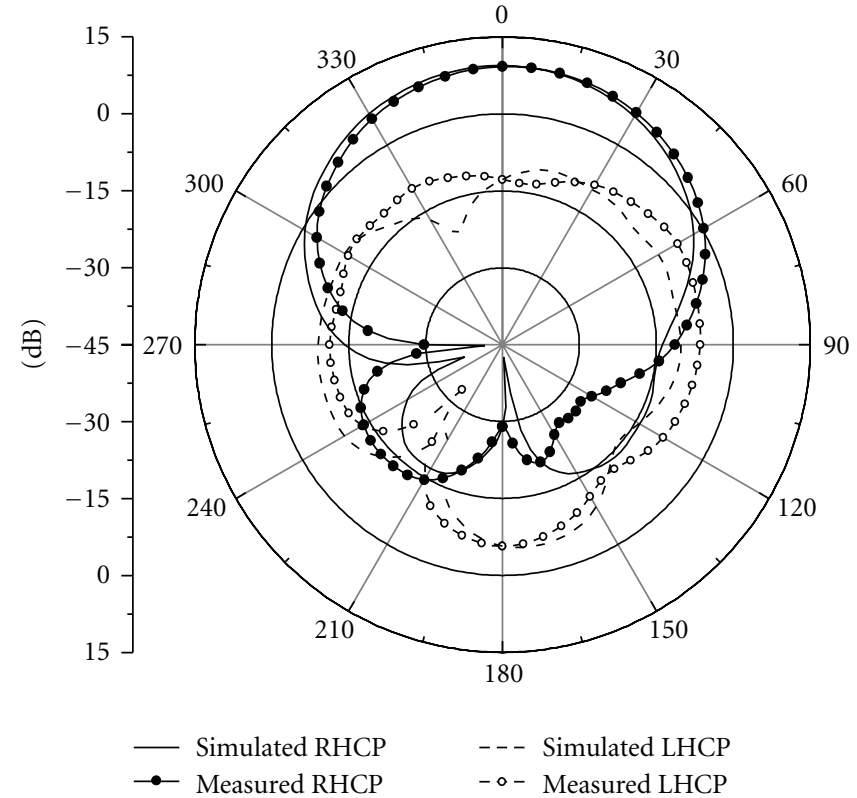

FIGURE 7: Simulated and measured radiation pattern at the center frequency $1.593 \mathrm{GHz}$.

optimized by using variable pitch, angle, and then the VSWR bandwidth can be tuned to coincide with the axial ratio bandwidth by changing a copper strip match stub size and position. Good antenna characteristics are achieved in the entire INMARSAT working band. The proposed antenna has many advantages such as simple structure, low-profile and easy to tune. It seems to be a well-suited antenna element candidate for maritime satellite communication applications.

\section{Acknowledgments}

This work has been supported by the National Science and Technology Support Project of China (no. 2012BAH36B01), the National Natural Science Foundation of China (no. 61071044), and the Applied Basic Research Programs of Ministry of Transportation of China (no. 2010-329-225-030). The authors are grateful to the Fundamental Research Funds for the Central Universities of China (No. 2011QN026) for providing financial assistance.

\section{References}

[1] J. D. Kraus, "Helical beam antennas," Electronics, vol. 20, pp. 109-111, 1947.

[2] H. Nakano, H. Takeda, T. Honma, H. Mimaki, and J. Yamauchi, "Extremely low-profile helix radiating a circularly polarized wave," IEEE Transactions on Antennas and Propagation, vol. 39, no. 6, pp. 754-757, 1991.

[3] C.-H. Chen, E. K. N. Yung, B.-J. Hu, and S.-L. Xie, "Axial mode helix antenna with exponential spacing," Microwave and Optical Technology Letters, vol. 49, no. 7, pp. 1525-1530, 2007.

[4] F. Yang, P. Zhang, C.-J. Guo, and J.-D. Xu, "Axial-mode elliptical helical antenna with variable pitch angle," Electronics Letters, vol. 44, no. 19, pp. 1103-1104, 2008. 
[5] H. T. Hui, K. Y. Chan, and E. K. N. Yung, "The low-profile hemispherical helical antenna with circular polarization radiation over a wide angular range," IEEE Transactions on Antennas and Propagation, vol. 51, no. 6, pp. 1415-1418, 2003.

[6] J. D. Kraus, "A 50-ohm input impedance for helical beam antennas," IEEE Transactions on Antennas and Propagation, vol. 25, no. 6, pp. 913-914, 1977.

[7] H. Nakano, K. Sato, H. Mimaki, and J. Yamauchi, "A long helical antenna wound on a dielectric rod," in Proceedings of the International Society for Asphalt Pavements, pp. 965-968, 2004.

[8] C. H. Chen, B. J. Hu, Z. H. Wu, and E. K. N. Yung, "A self-matching hemispherical helical antenna," in Proceedings of the IEEE Symposium on Antennas and Propagation, pp. 47094712, 2006.

[9] A. R. Djordjević, A. G. Zajić, M. M. Ilić, and G. L. Stüber, "Optimization of helical antennas," IEEE Antennas and Propagation Magazine, vol. 48, no. 6, pp. 107-115, 2006.

[10] H. E. King, J. L. Wong, and E. H. Newman, "Helical antennas," in Antenna Engineering Handbook, J. L. Volakis, Ed., McGrawHill, New York, NY, USA, 2007. 

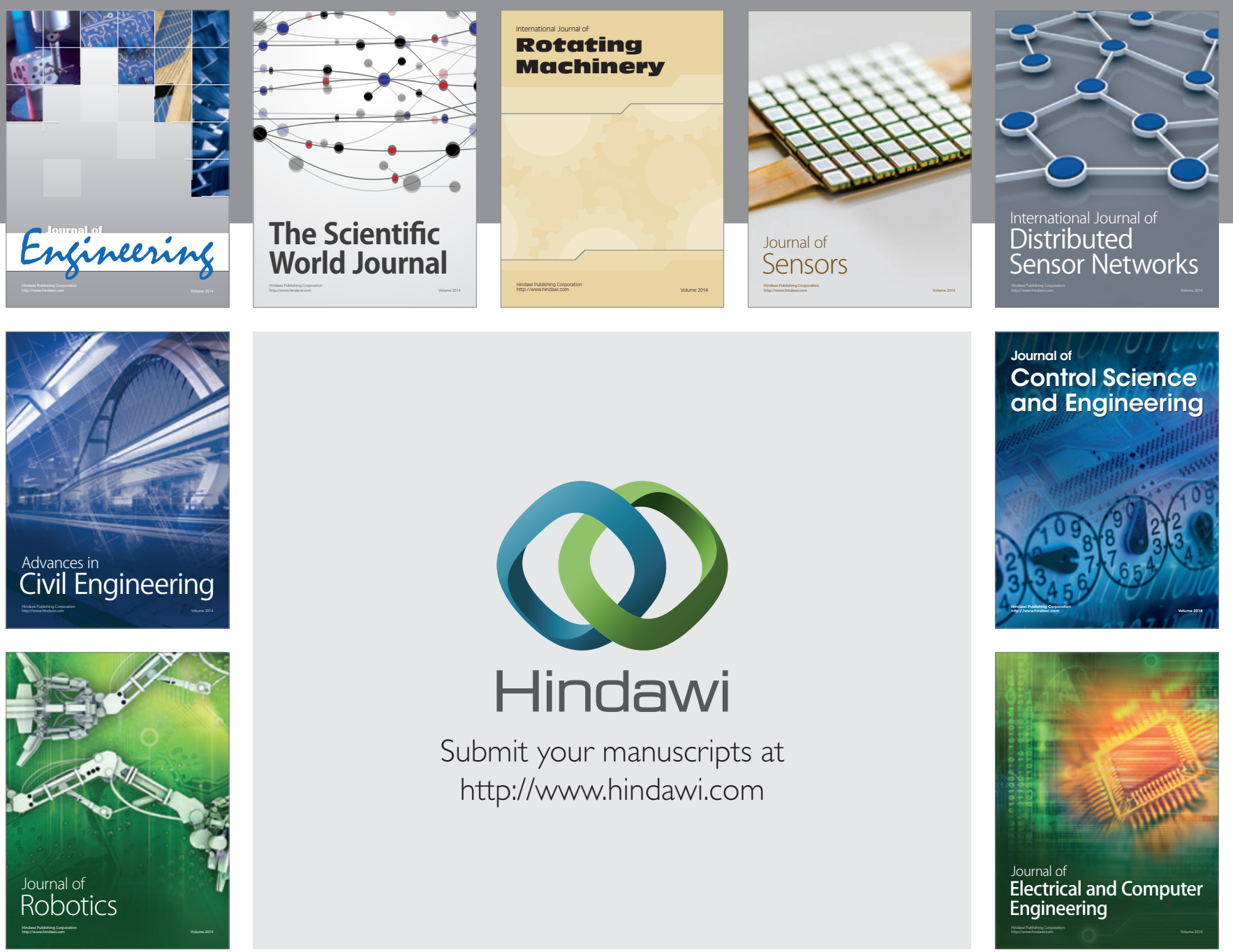

Submit your manuscripts at

http://www.hindawi.com
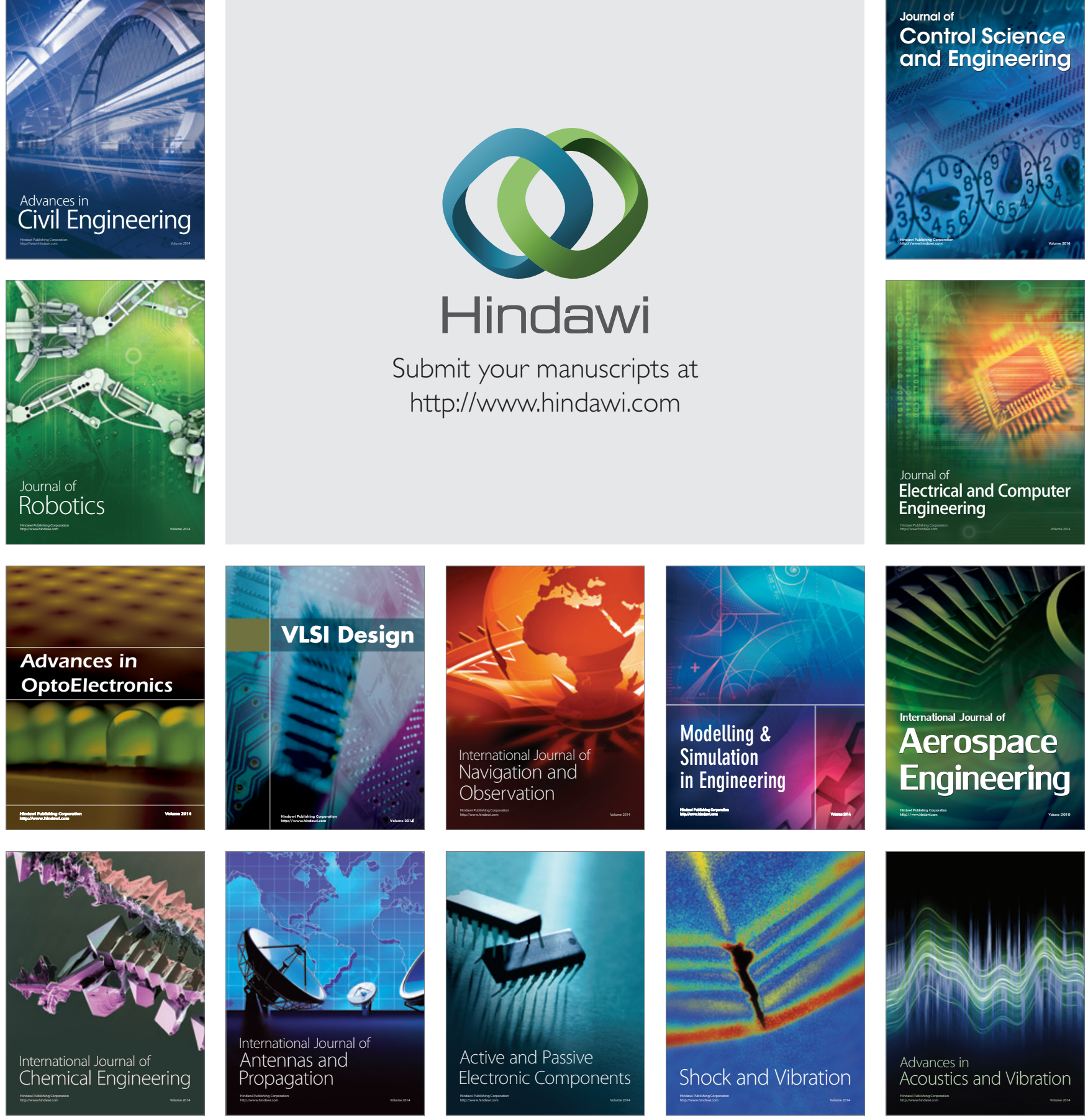